\title{
The Gut-Brain Axis: The Missing Link in Depression
}

\author{
Alper Evrensel, Mehmet Emin Ceylan \\ Department of Psychiatry, Uskudar University, Istanbul, Turkey
}

\begin{abstract}
The gut microbiota is essential to human health and the immune system and plays a major role in the bidirectional communication between the gut and the brain. Based on evidence, the gut microbiota is associated with metabolic disorders such as obesity, diabetes mellitus and neuropsychiatric disorders such as schizophrenia, autistic disorders, anxiety disorders and major depressive disorders. In the past few years, neuroscientific research has shown the importance of the microbiota in the development of brain systems. Recent studies showed that the microbiota could activate the immune and central nervous systems, including commensal and pathogenic microorganisms in the gastrointestinal tract. Gut microorganisms are capable of producing and delivering neuroactive substances such as serotonin and gamma-aminobutyric acid, which act on the gut-brain axis. Preclinical research in rodents suggested that certain probiotics have antidepressant and anxiolytic activities. Effects may be mediated via the immune system or neuroendocrine systems. Herein, we present the latest literature examining the effects of the gut microbiota on depression
\end{abstract}

KEY WORDS: Depression; Probiotics; Microbiota

\section{INTRODUCTION}

The effects of the gut microbiota on the immune system, brain development and behavior have attracted attention in recent years. Over $90 \%$ of the more than 4,000 articles on microbiota were published in PubMed in the last 5 years. ${ }^{1)}$ Presumably, microorganisms living in the intestine are in contact with the gut epithelial and immune system cells, and through this contact are involved in the development of many neuropsychiatric and metabolic disorders, particularly autoimmune diseases. Clinical observations and animal trials have revealed much evidence of a strong link between the gut and the brain, which is established during the intrauterine period and the influence of which continues throughout the life of an individual. ${ }^{2)}$

The term microbiome refers to all microorganisms and their genetic material living in the body, and the term microbiota refers to populations of microorganisms present in the body's various ecosystems (for example, the gut microbiota and skin microbiota). ${ }^{1)}$ There are $10^{14}$ microorganisms in the gut, which is 10 -fold greater than the

Received: March 1, 2015 / Revised: April 1, 2015

Accepted: June 29, 2015

Address for correspondence: Alper Evrensel, MD

Nispetiye cad. no:19 Beşiktaş, Istanbul 34330, Turkey

Tel: +90-2122701292, Fax: +90-2122701719

E-mail: alperevrensel@gmail.com number of human cells. These microorganisms also contain 150-fold more genes than the human genome.,

Similar to the Human Genome Project, another study, the Human Microbiome Project (HMP), is being conducted. ${ }^{5)}$ The aim of this project is to understand the diversity of the microbiome and microbiotas in various anatomical regions and determine the roles of microorganisms in health and disease. HMP is supported by the National Institutes of Health (NIH); in 2014, more than one million US dollars (USD) in resources was transferred to the project. ${ }^{6}$ )

Improvements in modern life such as antimicrobial treatments, vaccinations, intensive use of disinfecting and cleaning products and dietary changes exert a deep and lasting impact on the microbiome. ${ }^{7)}$ Changes in the gut microbiota may cause Clostridium difficile infection, ${ }^{8)}$ irritable bowel syndrome (IBS), ${ }^{9)}$ pathogen colonization (e.g., vancomycin-resistant Enterococcus), ${ }^{10)}$ autoimmune and allergic diseases, ${ }^{11)}$ obesity and metabolic disorders, ${ }^{12)}$ and neuropsychiatric disorders such as autism. ${ }^{13)}$

In this review we explain the pathophysiological mechanisms of the gut-brain axis, show the possible impact of the gut microbiota on depression, and inform clinicians of the latest scientific developments in this field.

(a) This is an Open-Access article distributed under the terms of the Creative Commons Attribution Non-Commercial License (http://creativecommons.org/licenses/by-nc/4.0) which permits unrestricted non-commercial use, distribution, and reproduction in any medium, provided the original work is properly cited. 


\section{MAIN SUBJECTS}

\section{Hygiene, Immunity, and the Gut Microbiota}

The hygiene hypothesis originated in 1989. According to this hypothesis, the reduced contact with microorganisms, especially during childhood, lifestyles away from nature and settlement mainly in large cities have resulted in an increased prevalence of allergic diseases. ${ }^{14)}$ Rook et al. ${ }^{15)}$ extended slightly this proposal and initiated the "old friends" hypothesis. According to this hypothesis, Homo sapiens have been evolving and interacting for thousands of years with friendly microorganisms in the body. Specifically, metabolites and nucleic acids produced by living microorganisms in the gut are transported into the human systemic circulation and lead to activation of inactive genes through epigenetic mechanisms, which can benefit health, lead to the development of various diseases and evolution. ${ }^{16,17)}$ (According to old friends hypothesis, Homo sapiens have been evolving and interacting for thousands of years with friendly microorganisms in the body.)

Effects of growing up in a more hygienic environment and changes in diet are mainly observed in developed countries, where the incidence of allergic diseases and autism is increasing. ${ }^{13)}$ The hygiene or "old friends" hypothesis may explain this phenomenon.

Various bacterial families - such as firmicutes, bacteroidetes, actinobacteria and proteobacteria - reside in the human intestine. ${ }^{1)}$ The human gut microbiota contains more than 1,000 species and over 7,000 subspecies. ${ }^{4)}$ Gut bacteria are able to produce active metabolites for human organ systems. For example; Lactobacillus and Bifidobacterium synthesize gamma-aminobutyric acid (GABA) from monosodium glutamate. ${ }^{18)}$ Escherichia coli, Bacillus and Saccharomyces produce norepinefrin; Candida, Streptococcus, Escherichia and Enterococcus produce serotonin, and Bacillus and Serratia produce dopamine. ${ }^{19)}$ When rats were given Bifidobacterium infantis orally, increased plasma tryptophan levels were observed. ${ }^{20)}$ Lactobacillus acidophilus increases the expression of cannabinoid receptors in the brainstem. ${ }^{21)}$ Conversely, germ-free (GF) rats had high plasma serotonin levels. ${ }^{22}$

The diversity of the gut microbiota can change under the influence of drugs, diet and stress. After 21 days of olanzapine administration in rodents, decreased proteobacteria and actinobacteria levels and increased firmicutes levels were observed. ${ }^{23)}$ Postnatal stress caused by the separation of rhesus monkeys from their mother changed the microbiota and decreased the Bifidobacte- rium and Lactobacillus levels. ${ }^{24)}$ Rats separated from their mother showed decreased fecal Lactobacillus levels on the third day; this effect was maintained for an extended period of time. $^{25)}$

The gut microbiota has been shown to play a critical role in innate and acquired immunity. ${ }^{12,26)}$ According to an article published in Science, microbiota diversity that develops during the perinatal period can have a lifelong effect on natural killer $\mathrm{T}$ lymphocytes. ${ }^{27)}$ Interaction between the microbiota and the gut mucosa regulates the production of several proinflammatory cytokines and chemokines, such as interleukin (IL)-8 and IL-1, IL-10 and transforming growth factor $\beta$ (TGF- $\beta)^{28)}$

Bacteria living in the gut interact with human cells. ${ }^{29)}$ This interaction occurs via one of the pattern recognition receptors (PRRs), namely the toll-like receptor (TLR). ${ }^{30,31)}$ Ten TLRs have been identified in the immune system. ${ }^{32)}$ These receptors are the first step in cytokine production pathways and are widely expressed in neurons. ${ }^{33,34)}$

Inflammatory cytokines such as interferon-alpha are known to cause depression and are blocked by antidepressants. $^{35,36)}$ The adenosine triphosphate (ATP)-sensitive P2X7 receptor (P2X7R) plays an important role in microglial activation caused by inflammation. The P2X7R antagonist brilliant blue $\mathrm{G}$ has anti-inflammatory and antidepressant effects in mice. ${ }^{37}$

In addition to their effects on monoamines, antidepressants exert their effects by suppressing inflammation via the potent immunoregulatory cytokine IL- $10{ }^{38)}$ Interestingly, probiotics also increase IL-10 levels. ${ }^{39)}$ When GF rats were given oral commensal bacteria, IL-10 synthesis and levels were increased. ${ }^{40)}$ Plasma IL-10 levels were increased in experimental animals when their offspring were given Lactobacillus rhamnosus GG. ${ }^{41)}$ During the development of the newborn, sudden changes occurring in the microbiota may have long-term consequences. ${ }^{42}$

The gut epithelium is the largest mucosal surface in the body. In healthy conditions, the tight junctions in the gut epithelium (occludin, adhesion molecule and zonula occludens) and mucus layer form a physical barrier to bacteria and foreign antigens. ${ }^{2)}$ Due to microdamage to the gut epithelium walls caused by changes in the microbiota and increased permeability of the gut epithelium, harmful substances produced by microorganisms enter the systemic circulation. ${ }^{13)}$ Bacterial proteins with neuroactive properties (p-cresol and 4-methylphenol) may enter the systemic circulation by disrupting the gut epithelial wall integrity. ${ }^{13)}$ Absorption into the circulation of pathogenic antigens can induce an immune response. ${ }^{43)}$ Studies with 
rodents showed that production of inflammatory cytokines increases with the impairment of gut permeability as bacterial lipopolysaccharides enter the systemic circulation and TLR4 and other TLRs are stimulated. ${ }^{44)}$

The gut microbiota has relationships with systems other than immunity. Subepithelial dendritic cells, one of the basic cells of the gut immune system, extend their dendrites through epithelial cells into the gut lumen and collect bacteria and their metabolites in the lumen. These materials are carried in lipoprotein vesicles, exosomes, which contain proteins, nucleic acids, sugars and lipids. This content is transferred from dendritic cells to T cells in the lymph nodes. Exosomes enter in the systemic circulation via the lymphatic system and blood and can reach the brain by passing through the blood-brain barrier. This process causes changes in the electrical layout of neuronal membranes. $^{45)}$

\section{Role of the Gut Microbiota in the Brain Functions}

The gastrointestinal system (GIS) is the largest immune organ. GIS-related products may be neuropathogenic. ${ }^{46)}$ Potential mechanisms influencing the microbiota of the central nervous system include changes in microbial content, ${ }^{22)}$ immune stimulation, neural pathways (via the vagus nerve), tryptophan metabolism, ${ }^{47)}$ gut hormonal response, ${ }^{48)}$ and bacterial metabolites. ${ }^{2)}$ Carbohydrate fermentation results in the formation of short-chain fatty acids, which affect brain functions by entering the systemic circulation. ${ }^{49-51)}$ The vagus nerve forms a direct connection between the brain and stomach. Hormonal, neuronal and bacterial changes in the bowel are transmitted to the brain via the vagus nerve. ${ }^{52-54)}$ The gut microbiota affects brain development and plasticity by secreting various neurotrophins and proteins, such as brain-derived neurotrophic factor (BDNF), synaptophysin and postsynaptic density (PSD)-95. ${ }^{55,56)}$

\section{The Role of the Microbiota in Depression}

The relationship between the microbiota and anxiety/depression has been studied mainly using animal models. Campylobacter jejuni administered orally to rats in subclinical doses led to anxiety-like behavior without an immune response. ${ }^{57)}$ Experimentally elevated hypothalamic-pituitary-adrenal (HPA) axis response and depression in GF rats can be reversed by administering a single bacterium, Bifidobacterium infantis ${ }^{58)}$ which is found predominantly in the neonatal intestinal tract, and probiotic drugs. This bacterium is defined as "psychobiotic" due to its antidepressant effects. ${ }^{59)}$
In the study by Desbonnet et al., ${ }^{58)}$ rat offspring separated from their mother were placed in two different groups and treated with Bifidobacterium infantis and citalopram (30 mg/kg in drinking water). Plasma cytokine levels, brain monoamine levels, central and peripheral HPA hormone levels were determined and depressive behaviors were measured using the forced swimming test. Separation from the mother caused a decrease in swimming in the forced swimming test and increased the immobility behavior, decreased norepinephrine levels in the brain, increased peripheral proinflammatory IL-6 secretion and corticotrophin-releasing factor mRNA levels in the amygdala. Probiotic therapy has led to a reversal of behavior problems and to the normalization of the immune response and norepinephrine levels in the brain. Based on these results, it was suggested that administration of Bifidobacterium infantis leads to relaxation in neural processes. In rats administered citalopram or bifidobacteria, no significant effects were observed on climbing behavior in the forced swimming test and no differences in corticosterone and cytokine concentrations were observed between the groups. ${ }^{58)}$

Connections between the microbiota and anxiety-related behaviors in rats have been shown in many studies. ${ }^{55,60,61)}$ Rats administered Lactobacillus helveticus and Bifidobacterium longum for 14 days showed low scores in anxiety tests. ${ }^{62)}$ In the double-blind placebo-controlled and randomized parallel group study by the same researchers using healthy volunteers, subjects took Lactobacillus helveticus R0052 and Bifidobacterium longum or placebo for 30 days. Psychiatric parameters were evaluated using various tests (Hopkins symptom checklist, hospital anxiety and depression scale, the perceived stress scale and coping checklist). The results showed psychological stress levels and urinary free cortisol levels were decreased in subjects who took the probiotics regularly. $^{63)}$

In another experiment, rats given Lactobacillus rhamnosus for 28 days showed a decline in both anxiety and depression scores. ${ }^{64)}$ The most significant results were observed in the experiment where anxiety-like behavior obtained with high-fat diet was prevented by the administration of Lactobacillus helveticus for 21 days; when the experiment was duplicated using IL-10-deficient rats, no changes in anxiety were observed. These results showed the role of the immune system in the gut-brain axis. ${ }^{65)}$ Administration of Lactobacillus farciminis to rats reduced the HPA axis response to stress (plasma adrenocorticotropic hormone [ACTH], corticosteroid levels and corti- 
cotropin-releasing hormone [CRH] levels). ${ }^{44)}$

In clinical trials, the Bacteroides family has been shown to be associated with depression. ${ }^{66)}$ Serum immunoglobulin (Ig)M and IgA antagonist lipopolysaccharide levels were increased. ${ }^{38)}$ In healthy individuals, milk fermented with probiotic bacteria showed a positive effect on the emotional centers. The study by Tillisch et al. ${ }^{67)}$ examined the relationship between probiotics and healthy brain functions using functional magnetic resonance imaging (fMRI). Berk et al. ${ }^{68)}$ reported that a chronic, low-level inflammatory condition is present in depression, which may be associated with gut permeability disorders. The microbiota has been suggested to be the key factor in the link between unhealthy diets and depression. ${ }^{69)}$

\section{Treatment Regulating the Gut Microbiota}

Although the interest in the microbiota and probiotic treatments has increased in recent years, the first probiotic treatment for depression was implemented in $1910{ }^{70)} \mathrm{A}$ prebiotic promotes the growth or survival of a particular gut microorganism; a probiotic is defined as oral or rectal administration of a particular microorganism. ${ }^{1)}$ In the United States, the annual market for nonprescription probiotics is estimated at one billion USD. ${ }^{71}$

Activated charcoal is used in the treatment of poisoning occurring after an overdose. Charcoal inhibits absorption from the intestine by binding to toxins. To reduce diarrhea, indigestion and bloating symptoms, tablets and capsules are used, which assists recovery of the GIS by binding to toxins secreted by the microbiota. ${ }^{72)}$

Activated charcoal is used successfully in fecal microbiota transplantation (FMT) for Clostridium difficile infection, IBS and inflammatory bowel disease (ulcerative colitis and Crohn's disease). ${ }^{8)}$ Recently, studies on its efficacy in the treatment of cardiometabolic and autoimmune disorders have increased. ${ }^{16,73)}$ Side-effects of probiotics and FMT are unlikely. The ideal approach is to restore the microbiota using probiotics without FMT.

\section{CONCLUSION}

The effects of the gut microbiota on human health will be an area of interest in neuroscience over the next 10 years. Several authors suggest microorganisms as a new group of drugs named "psychomicrobiotics" for the treatment of psychiatric disorders. ${ }^{72)}$ Recent research has suggested that the gut microbiota has an influence on mood. Poor diet is a risk factor for depression; thus, a healthy diet may prevent depression. Regulation of the gut microbiota using diet, probiotics and FMT may have important benefits for preventing and treating depression. The gut-brain axis could aid in understanding and treating neuropsychiatric disorders, especially depression. ${ }^{74)}$

\section{REFERENCES}

1. Khanna S, Tosh PK. A clinician's primer on the role of the microbiome in human health and disease. Mayo Clin Proc 2014;89:107-114.

2. Borre YE, O'Keeffe GW, Clarke G, Stanton C, Dinan TG, Cryan JF. Microbiota and neurodevelopmental windows: implications for brain disorders. Trends Mol Med 2014; 20:509-518.

3. de Vos WM, de Vos EA. Role of the intestinal microbiome in health and disease: from correlation to causation. Nutr Rev 2012;70 Suppl 1:S45-S56.

4. Lozupone CA, Stombaugh JI, Gordon JI, Jansson JK, Knight R. Diversity, stability and resilience of the human gut microbiota. Nature 2012;489:220-230.

5. Jumpstart Consortium Human Microbiome Project Data Generation Working Group. Evaluation of $16 \mathrm{~S}$ rDNA-based community profiling for human microbiome research. PLoS One 2012;7:e39315.

6. Reardon S. Gut-brain link grabs neuroscientists. Nature 2014;515:175-177.

7. Flint HJ. The impact of nutrition on the human microbiome. Nutr Rev 2012;70 Suppl 1:S10-S13.

8. Brandt LJ. American Journal of Gastroenterology Lecture: Intestinal microbiota and the role of fecal microbiota transplant (FMT) in treatment of $C$. difficile infection. Am $J$ Gastroenterol 2013;108:177-185.

9. O'Mahony L, McCarthy J, Kelly P, Hurley G, Luo F, Chen $\mathrm{K}$, et al. Lactobacillus and bifidobacterium in irritable bowel syndrome: symptom responses and relationship to cytokine profiles. Gastroenterology 2005;128:541-551.

10. Tosh PK, McDonald LC. Infection control in the multidrugresistant era: tending the human microbiome. Clin Infect Dis 2012;54:707-713.

11. Martínez I, Lattimer JM, Hubach KL, Case JA, Yang J, Weber CG, et al. Gut microbiome composition is linked to whole grain-induced immunological improvements. ISME J 2013; 7:269-280.

12. Bäckhed F, Manchester JK, Semenkovich CF, Gordon JI. Mechanisms underlying the resistance to diet-induced obesity in germ-free mice. Proc Natl Acad Sci USA 2007; 104:979-984.

13. Hornig M. The role of microbes and autoimmunity in the pathogenesis of neuropsychiatric illness. Curr Opin Rheumatol 2013;25:488-795.

14. Strachan DP. Hay fever, hygiene, and household size. BMJ 1989;299:1259-1260.

15. Rook GA, Martinelli R, Brunet LR. Innate immune responses to mycobacteria and the downregulation of atopic responses. Curr Opin Allergy Clin Immunol 2003;3:337-342.

16. Rook GA. 99th Dahlem conference on infection, inflammation and chronic inflammatory disorders: darwinian medicine and the 'hygiene' or 'old friends' hypothesis. Clin Exp Immunol 2010;160:70-79.

17. Rook GA, Lowry CA, Raison CL. Hygiene and other early childhood influences on the subsequent function of the immune system. Brain Res 2015;1617:47-62.

18. Barrett E, Ross RP, O'Toole PW, Fitzgerald GF, Stanton C. 
$\gamma$-Aminobutyric acid production by culturable bacteria from the human intestine. J Appl Microbiol 2012;113: 411-417.

19. Lyte M. Probiotics function mechanistically as delivery vehicles for neuroactive compounds: Microbial endocrinology in the design and use of probiotics. Bioessays 2011;33: 574-581.

20. Desbonnet L, Garrett L, Clarke G, Bienenstock J, Dinan TG. The probiotic Bifidobacteria infantis: An assessment of potential antidepressant properties in the rat. J Psychiatr Res 2008;43:164-174.

21. Muccioli GG, Naslain D, Bäckhed F, Reigstad CS, Lambert DM, Delzenne NM, et al. The endocannabinoid system links gut microbiota to adipogenesis. Mol Syst Biol 2010;6:392.

22. Collins SM, Bercik P. The relationship between intestinal microbiota and the central nervous system in normal gastrointestinal function and disease. Gastroenterology 2009;136:2003-2014.

23. Davey KJ, O'Mahony SM, Schellekens H, O'Sullivan O, Bienenstock J, Cotter PD, et al. Gender-dependent consequences of chronic olanzapine in the rat: effects on body weight, inflammatory, metabolic and microbiota parameters. Psychopharmacology (Berl) 2012;221:155-169.

24. Bailey MT, Coe CL. Maternal separation disrupts the integrity of the intestinal microflora in infant rhesus monkeys. Dev Psychobiol 1999;35:146-155.

25. O'Mahony SM, Marchesi JR, Scully P, Codling C, Ceolho AM, Quigley EM, et al. Early life stress alters behavior, immunity, and microbiota in rats: implications for irritable bowel syndrome and psychiatric illnesses. Biol Psychiatry 2009;65:263-267.

26. Round JL, O'Connell RM, Mazmanian SK. Coordination of tolerogenic immune responses by the commensal microbiota. J Autoimmun 2010;34:J220-J225.

27. Olszak T, An D, Zeissig S, Vera MP, Richter J, Franke A, et al. Microbial exposure during early life has persistent effects on natural killer $T$ cell function. Science 2012; 336:489-493.

28. Neish AS. Microbes in gastrointestinal health and disease. Gastroenterology 2009;136:65-80.

29. Dinan TG, Quigley EM. Probiotics in the treatment of depression: science or science fiction? Aust $N Z J$ Psychiatry 2011;45:1023-1025.

30. Carvalho FA, Aitken JD, Vijay-Kumar M, Gewirtz AT. Toll-like receptor-gut microbiota interactions: perturb at your own risk! Annu Rev Physiol 2012;74:177-198.

31. Lucas K, Maes M. Role of the Toll Like receptor (TLR) radical cycle in chronic inflammation: possible treatments targeting the TLR4 pathway. Mol Neurobiol 2013;48:190204.

32. Takeuchi O, Akira S. Pattern recognition receptors and inflammation. Cell 2010;140:805-820.

33. McKernan DP, Dennison U, Gaszner G, Cryan JF, Dinan TG. Enhanced peripheral toll-like receptor responses in psychosis: further evidence of a pro-inflammatory phenotype. Transl Psychiatry 2011;1:e36.

34. McCusker RH, Kelley KW. Immune-neural connections: how the immune system's response to infectious agents influences behavior. J Exp Biol 2013;216:84-98.

35. Udina M, Castellví P, Moreno-España J, Navinés R, Valdés $\mathrm{M}$, Forns $\mathrm{X}$, et al. Interferon-induced depression in chronic hepatitis C: a systematic review and meta-analysis. J Clin Psychiatry 2012;73:1128-1138.

36. McNutt MD, Liu S, Manatunga A, Royster EB, Raison CL, Woolwine BJ, et al. Neurobehavioral effects of interferon- a in patients with hepatitis-C: symptom dimensions and responsiveness to paroxetine. Neuropsychopharmacology 2012;37:1444-1454.

37. Ma M, Ren Q, Zhang JC, Hashimoto K. Effects of Brilliant Blue $G$ on serum tumor necrosis factor-a levels and depression-like behavior in mice after lipopolysaccharide administration. Clin Psychopharmacol Neurosci 2014;12: 31-36.

38. Maes M, Kenis G, Kubera M, De Baets M, Steinbusch H, Bosmans E. The negative immunoregulatory effects of fluoxetine in relation to the cAMP-dependent PKA pathway. Int Immunopharmacol 2005;5:609-618.

39. Levkovich T, Poutahidis T, Smillie C, Varian BJ, Ibrahim YM, Lakritz JR, et al. Probiotic bacteria induce a 'glow of health'. PLoS One 2013;8:e53867.

40. Macpherson AJ, Uhr T. Gut flora--mechanisms of regulation. Eur J Surg Suppl 2002;(587):53-57.

41. Kopp MV, Goldstein M, Dietschek A, Sofke J, Heinzmann A, Urbanek R. Lactobacillus $G G$ has in vitro effects on enhanced interleukin-10 and interferon-gamma release of mononuclear cells but no in vivo effects in supplemented mothers and their neonates. Clin Exp Allergy 2008;38: 602-610.

42. Costello EK, Stagaman K, Dethlefsen L, Bohannan BJ, Relman DA. The application of ecological theory toward an understanding of the human microbiome. Science 2012; 336:1255-1262.

43. Fetissov SO, Déchelotte P. The new link between gut-brain axis and neuropsychiatric disorders. Curr Opin Clin Nutr Metab Care 2011;14:477-482.

44. Ait-Belgnaoui A, Durand H, Cartier C, Chaumaz G, Eutamene H, Ferrier L, et al. Prevention of gut leakiness by a probiotic treatment leads to attenuated HPA response to an acute psychological stress in rats. Psychoneuroendocrinology 2012;37:1885-1895.

45. Smythies LE, Smythies JR. Microbiota, the immune system, black moods and the brain-melancholia updated. Front Hum Neurosci 2014;8:720.

46. Severance EG, Gressitt KL, Stallings CR, Origoni AE, Khushalani S, Leweke FM, et al. Discordant patterns of bacterial translocation markers and implications for innate immune imbalances in schizophrenia. Schizophr Res 2013;148:130-137.

47. O'Mahony SM, Clarke G, Borre YE, Dinan TG, Cryan JF. Serotonin, tryptophan metabolism and the brain-gutmicrobiome axis. Behav Brain Res 2015;277:32-48.

48. Wren AM, Bloom SR. Gut hormones and appetite control. Gastroenterology 2007;132:2116-2130.

49. Macfarlane S, Macfarlane GT. Regulation of short-chain fatty acid production. Proc Nutr Soc 2003;62:67-72.

50. Macfabe DF. Short-chain fatty acid fermentation products of the gut microbiome: implications in autism spectrum disorders. Microb Ecol Health Dis 2012;23. doi: 10.3402/ mehd.v23i0.19260.

51. Kimura I, Ozawa K, Inoue D, Imamura T, Kimura K, Maeda $\mathrm{T}$, et al. The gut microbiota suppresses insulin-mediated fat accumulation via the short-chain fatty acid receptor GPR43. Nat Commun 2013;4:1829.

52. Wang X, Wang BR, Zhang XJ, Xu Z, Ding YQ, Ju G. Evidences for vagus nerve in maintenance of immune balance and transmission of immune information from gut to brain in STM-infected rats. World $J$ Gastroenterol 2002;8:540-545.

53. Borovikova LV, Ivanova S, Zhang M, Yang H, Botchkina GI, Watkins LR, et al. Vagus nerve stimulation attenuates 
the systemic inflammatory response to endotoxin. Nature 2000;405:458-462.

54. Perez-Burgos A, Wang B, Mao YK, Mistry B, McVey Neufeld KA, Bienenstock J, et al. Psychoactive bacteria Lactobacillus rhamnosus (JB-1) elicits rapid frequency facilitation in vagal afferents. Am J Physiol Gastrointest Liver Physiol 2013;304:G211-G220.

55. Diaz Heijtz R, Wang S, Anuar F, Qian Y, Björkholm B, Samuelsson A, et al. Normal gut microbiota modulates brain development and behavior. Proc Natl Acad Sci U S A 2011;108:3047-3052.

56. Douglas-Escobar M, Elliott E, Neu J. Effect of intestinal microbial ecology on the developing brain. JAMA Pediatr 2013;167:374-379.

57. Lyte M, Varcoe JJ, Bailey MT. Anxiogenic effect of subclinical bacterial infection in mice in the absence of overt immune activation. Physiol Behav 1998;65:63-68.

58. Desbonnet L, Garrett L, Clarke G, Kiely B, Cryan JF, Dinan TG. Effects of the probiotic Bifidobacterium infantis in the maternal separation model of depression. Neuroscience 2010;170:1179-1188.

59. Dinan TG, Stanton C, Cryan JF. Psychobiotics: a novel class of psychotropic. Biol Psychiatry 2013;74:720-726.

60. Neufeld KA, Kang N, Bienenstock J, Foster JA. Effects of intestinal microbiota on anxiety-like behavior. Commun Integr Biol 2011;4:492-494.

61. Clarke G, Grenham S, Scully P, Fitzgerald P, Moloney RD, Shanahan $\mathrm{F}$, et al. The microbiome-gut-brain axis during early life regulates the hippocampal serotonergic system in a sex-dependent manner. Mol Psychiatry 2013;18:666-673.

62. Messaoudi M, Lalonde R, Violle N, Javelot H, Desor D, Nejdi A, et al. Assessment of psychotropic-like properties of a probiotic formulation (Lactobacillus helveticus R0052 and Bifidobacterium longum R0175) in rats and human subjects. Br J Nutr 2011;105:755-764.

63. Messaoudi M, Violle N, Bisson JF, Desor D, Javelot H, Rougeot C. Beneficial psychological effects of a probiotic formulation (Lactobacillus helveticus R0052 and Bifidobacterium longum R0175) in healthy human volunteers. Gut Microbes 2011;2:256-261.
64. Bravo JA, Forsythe P, Chew MV, Escaravage E, Savignac $\mathrm{HM}$, Dinan TG, et al. Ingestion of Lactobacillus strain regulates emotional behavior and central GABA receptor expression in a mouse via the vagus nerve. Proc Natl Acad Sci U S A 2011;108:16050-16055.

65. Ohland CL, Kish L, Bell H, Thiesen A, Hotte N, Pankiv E, et al. Effects of Lactobacillus helveticus on murine behavior are dependent on diet and genotype and correlate with alterations in the gut microbiome. Psychoneuroendocrinology 2013;38:1738-1747.

66. Naseribafrouei A, Hestad K, Avershina E, Sekelja M, Linløkken A, Wilson $\mathrm{R}$, et al. Correlation between the human fecal microbiota and depression. Neurogastroenterol Motil 2014;26:1155-1162.

67. Tillisch K, Labus J, Kilpatrick L, Jiang Z, Stains J, Ebrat $\mathrm{B}$, et al. Consumption of fermented milk product with probiotic modulates brain activity. Gastroenterology 2013; 144:1394-1401.

68. Berk M, Williams LJ, Jacka FN, O'Neil A, Pasco JA, Moylan S, et al. So depression is an inflammatory disease, but where does the inflammation come from? BMC Med 2013;11:200.

69. Dash S, Clarke G, Berk M, Jacka FN. The gut microbiome and diet in psychiatry: focus on depression. Curr Opin Psychiatry 2015;28:1-6.

70. Phillips JGP. The treatment of melancholia by the lactic acid bacillus. Br J Psychiatry 1910;56:422-431.

71. Vanderhoof JA, Young R. Probiotics in the United States. Clin Infect Dis 2008;46 Suppl 2:S67-S72.

72. Fond G, Boukouaci W, Chevalier G, Regnault A, Eberl G, Hamdani N, et al. The "psychomicrobiotic": Targeting microbiota in major psychiatric disorders: a systematic review. Pathol Biol (Paris) 2015;63:35-42.

73. Smits LP, Bouter KE, de Vos WM, Borody TJ, Nieuwdorp M. Therapeutic potential of fecal microbiota transplantation. Gastroenterology 2013;145:946-953.

74. Evrensel A, Ceylan ME. Gut-brain axis: the role of gut microbiota in the psychiatric disorders. Curr Approach Psychiatry 2015;7:461-472. 